\title{
Machine Translation and Translation Theory
}

\author{
Christa Hauenschild and Susanne Heizmann (editors) \\ (University of Hildesheim)
}

\author{
Berlin: Mouton de Gruyter (Text, \\ translation, computational processing, \\ edited by Annely Rothkegel and John \\ Laffling), 1997, xiv+266 pp; hardbound, \\ ISBN 3-11-015486-2, DM 168.00
}

\section{Reviewed by \\ Frank Van Eynde \\ University of Leuven}

Machine Translation and Translation Theory is a collection of 14 papers, most of which were read at the Second International Workshop on Machine Translation and Translation Theory (Hildesheim University, September 1994). The aim of both the workshop and the book is to build a bridge between research on machine translation and research on human translation and interpreting. According to the editors, these fields have ignored one another far too long and would gain from closer mutual links. Just what those links might be is explored in three groups of papers.

The first group concerns aspects of human translation and interpreting that are argued to be of interest for machine translation. It includes contributions on the modeling of simultaneous interpreting (Barbara Moser-Mercer), on the teaching of consecutive interpreting (Hans Hönig), on the role of text functions (expressive, appellative, etc.) in the translation of titles and headings (Christiane Nord), on the importance of cultural specifics in the translation of job applications (Heidrun Gerzymisch-Arbogast), and on the role of information structure in translation (Monika Doherty). Of these five, however, there are only two that make an effort to spell out the relevance of their findings for MT. In "Using text mappings in teaching consecutive interpreting" (pp. 20-34) Hans Hönig pleads for the importation into MT of a technique that he uses for teaching consecutive interpreting. It consists in the mapping of a text onto a cognitive macrostructural scene that specifies the communicative intentions and strategies of the speaker. Knowing the latter is of considerable help in human interpreting, and is therefore likely to be useful for machine interpreting as well. Unfortunately, since speakers seldom make their intentions explicit, the macrostructural scenes have to be provided manually to the translation system, presumably in a training or preediting phase, and on the specifics of this training phase the author is very brief: "If I have understood the literature correctly, a connectionist, self-adaptive system will be most suitable for the necessary training, or at least a hybrid model" (p. 33). Equally brief are the comments on the feasibility of this enterprise, but with the insistence on the importance of macrostructural properties of texts the author strikes a chord that reverberates throughout the entire volume.

Monika Doherty's "Textual garden paths-Parametrized obstacles to target language adequate translations" (pp. 69-89) highlights the role of a text's information structure and discusses the articulation of this structure in terms of clauses and the links between them, as expressed by, for example, punctuation. She points out some salient differences in this respect between German and English, and provides an interesting discussion of the information structure within clauses. Assigning integers that reflect the informational weight of individual constituents ( 1 for the highest weight 
and 3 for the lowest), the author argues that the most natural translation of English verb-second structures, as in $\mathrm{S}(3) \mathrm{V}(1)$ Adjunct(2), into German verb-final structures is not $S(3) \operatorname{Adj}(2) \mathrm{V}(1)$, but rather $\operatorname{Adj}(2) S(3) \mathrm{V}(1)$ : "The preferred version is the structurally more 'balanced' translation version which places the lowest value between the higher ones" (p. 79). The proposal lacks formal precision and generality, but it provides an interesting illustration of how focus theories might be exploited in MT.

The second group of papers consists of studies of human translation that are oriented towards MT. Three of them report on work that was carried out in the framework of Verbmobil, a German project that aims at the development of a speech-to-speech translation system for appointment-scheduling dialogues from German and Japanese into English; see Wahlster (1993) and Kay, Gawron, and Norvig (1994). An important property of the interpreting in Verbmobil is that it is consecutive: the automatic interpreter waits for the first speaker to finish an utterance, then translates it (via English) into the other speaker's language, and only then does the latter take his or her turn: Simulating this scenario in human face-to-face translation, Birgit Apfelbaum and Cecilia Wadensjö draw attention to the fact that the role of the interpreter in such a setting is much more active than one might expect: interpreters do not just translate what they hear, but often coordinate the conversation and sometimes even initiate an exchange with one of the speakers, for instance, for clarification. This claim is substantiated with detailed empirical evidence, but what it implies for MT is not spelled out.

Somewhat more informative in this respect are the two studies described in the paper by Birte Prahl and Susanne Petzolt. The first study concerns the translation of a text that is not fully comprehensible, due to the absence of a situational context. Comparing the ways in which expert translators and translation students deal with such texts, the authors argue that the only sensible strategy is to preserve the degree of ambiguity of the SL text, as the experts do, and to resist the temptation to make the TL text more informative or less ambiguous than the original, as less-experienced translators tend to do.

The second study concerns the translation of utterances in typical Verbmobil scenarios. In that case, the SL text may be equally underspecified and ambiguous, but given the conventions of appointment-scheduling dialogues, the missing information can and should be inferred from what the authors call "standard assumptions" or "defaults." They also discuss the factors that should be taken into account when choosing an ambiguity-preserving or an ambiguity-resolving strategy, but provide no detail as to how the latter could be achieved.

The third contribution in this series concerns the automatic interpeting of dialogue acts. Its author, Susanne Jekat, claims that-for the purpose of translating appointmentscheduling dialogues-translational equivalence consists of two parts, i.e., the discourse referents that are directly related to the purpose of the dialogue (dates and hours of the day) and the dialogue acts that are realized by the consecutive turns in the dialogue, such as greeting, proposal of a date, rejection of a date, confirmation, etc. The text convincingly demonstrates the importance of the latter, but is rather vague on the issue of what the relevant dialogue acts are and especially on how they can be identified.

Besides the Verbmobil contributions, the second group includes two papers on lexical mismatches between languages. In "Compensation" (pp. 157-173), Louis des Tombe discusses, among others, the relation between the English cousin, which is sex-neutral, and the French cousin/cousine, which is invariably sex-specific. Such mismatches lead to translation problems whose solution cannot easily be cast in the mold of a strictly compositional treatment. As an alternative, the author proposes a treat- 
ment that involves the computation of differences in meaning between the imperfectly matching elements and the consistent use of the most specific terms, as long as they are not incompatible with the information in the rest of the text.

In "Interlingual strategies in translation" (pp. 175-190), Peter Pause discusses the lexical mismatches that result from the fact that the expression of motion, path, and manner tends to be packaged differently in Romance and Germanic verb phrases. For example, whereas in the German hinüberschwimmen, motion and manner are expressed by the verb schwimmen ('swim') and path by its separable prefix hinüber ('across'), French tends to package motion and path in the verb, leaving the expression of manner to an adverb or a gerund, as in traverser en nageant ('cross by swimming'). In order to model the translation in such cases, the author maps the language-specific words onto interlingual semantic representations and defines preference rules that operate on the latter.

While clearly different in the choice of examples and in the proposals on how to treat them, Louis des Tombe and Peter Pause share the assumption that translation involves a certain amount of inferencing on the basis of semantic representations. Moreover, in contrast to the previously mentioned contributions, they do not shy away from the task of spelling out in some formal detail how such inferencing could be done.

The papers of the third group present approaches to machine translation that take some inspiration from studies on human translation. Two of them are based on work for Verbmobil. "The translation objective in automatic dialogue interpreting" (pp. 193210) by Birte Schmitz can be read as a sequel to the paper by Susanne Jekat, for it is indeed in this contribution that Jekat's rather vague claims about dialogue acts get a more specific elaboration, to the point where implementation becomes a distinct possibility, at least in SL analysis. As the author herself remarks, the question of how TL generation can cope with this fairly abstract type of representation is still open. The contribution by Jan Amtrup, "Perspectives for incremental MT with charts" (pp. 211222 ), is more concerned with processing issues. As the title suggests, the author extends the incremental character of chart-based parsing to transfer and motivates this move, among others, with the observation that it provides a better model of the way in which human interpreting takes place. As a test case for his approach, he discusses some well-known cases of head switching, as in the English translation of the German adverb gerne ('like').

The third contribution in this group presents some of the research that is carried out in the framework of a similar but distinct project, i.e., interpreting telephony (Japan, U.S.A., Germany). It aims at the automatic translation of Japanese and English utterances in bilingual telephone conversations, more specifically in conference-registration calls. A crucial part of the system is the module that manages the dialogue interaction and processes each new utterance in context. It is presented at some length in Susann LuperFoy's "Discourse processing for voice-to-voice machine translation" (pp. 223250). In contrast to Verbmobil, which takes its inspiration in this field from speech-act theory, relevance theory, and functional approaches to text linguistics, interpreting telephony draws on formal approaches, such as file-change semantics and discourse representation theory. The article presupposes some familiarity with the latter and focuses on a number of extensions and modifications that have been found necessary in order to make these theories "work" in the interpreting telephony context. The most important of these extensions concern the segmentation of a discourse into units that correspond to speech acts and the facilitation of incremental interpretation and nonmonotonic updating of the discourse model.

Rather fittingly, the book closes with a contribution on the evaluation of translation 
systems. Margaret King's "Evaluating translation" (pp. 251-263) is actually an exercise in metaevaluation, for the object of her evaluation is not any (group of) MT systems, but rather the criteria in terms of which MT systems are commonly evaluated. After reviewing those that were employed by the Automatic Language Processing Advisory Committee (1966) and those that were designed in the early 1990s on behalf of ARPA, a major U.S. funding agency, the author concludes that translation quality cannot be defined in the abstract, mainly because there is no such thing as "the correct translation," and pleads for an evaluation that explicitly takes into account the specific purpose for which a translation has been made.

Now that I have gone through the individual papers, the obvious question is how much they contribute to the overall goal of the book, i.e., to help build a bridge between MT and translation theory. On this account the result is somewhat underwhelming, as the editors themselves seem to admit: "In view of the fact that only first stepping stones have been laid on this bridge-building site, this book cannot be regarded as a systematic and comprehensive collection but rather as a glimpse of the potential inherent in this interdisciplinary enterprise" (p. vii). There is, of course, a world of difference between building a bridge and providing a glimpse of its potential, and readers who are content with such glimpses will certainly find the entire book stimulating and inspiring, but those who are looking for some more solid and formally explicit proposals will probably want to limit their attention to the papers of the third group and some of the second group.

\section{References}

Kay, Martin, Jean Mark Gawron, and Peter Norvig. 1994. Verbmobil: A translation system for face-to-face dialogue. Stanford: Center for the Study of Language and Information.

Wahlster, Wolfgang. 1993. Verbmobil: Translation of face-to-face dialogs. Proceedings of Machine Translation Summit $I V$, Kobe, Japan.

Frank Van Eynde is associate professor at the University of Leuven and head of its Centre for Computational Linguistics. He has authored and edited various publications on machine translation, including the book Linguistic Issues in Machine Translation (London: Pinter Publishers, 1993). He is a member of the editorial boards of the journal Machine Translation and of the book series Studies in Machine Translation and Natural Language Processing. Van Eynde's address is: Centre for Computational Linguistics, University of Leuven, Maria-Theresiastraat 21, 3000 Leuven, Belgium; e-mail: frank.vaneynde@ccl.kuleuven.ac.be; http://www.ccl.kuleuven.ac.be/ frank 